\title{
Septoplasty Outcome Using Snot- 22 Questionnaire Study
}

\author{
*Dr. H.S.Satish, **Dr.K.T.Sreedhar \\ *Prof \& HOD, ** Post Graduate.
}

\begin{abstract}
Objective: Sino Nasal Outcome Test-22 (SNOT-22) Questionnaire study was done to analyse the effectiveness of septoplasty surgery by noting the improvement of nasal symptoms \& general quality of life( $Q O L)$ by means of SNOT-22 questionnaire given to patients before the surgery and 8 weeks after the surgery. Analysis of effectiveness of septoplasty is usually done by noting pre and post operative symptoms.

Study Design; This is a prospective study and our data analysis consisted originally of 76 patients of which 70 patients answered the SNOT-22 questions both preoperatively and postoperatively. The age group of the patients was between 18 to 55 years.

Results; 60 patients( $86 \%$ of the patients) who had mild to moderate symptoms(with SNOT-22 scoring < 40 points), and also young patients (<30 years) showed much improvement with the symptoms with relief from nasal obstruction after surgery. In the post operative SNOT-22, the need to blow nose, sneezing, running nose, nasal obstruction, loss of smell or taste, post nasal discharge, facial pain/pressure, difficulty in falling asleep, waking up at night lack of good night's sleep, wake up tired, reduced productivity and embarrassed improved significantly.
\end{abstract}

Conclusion: Evaluation of symptoms \& findings in the patient is essential in deciding whether surgery or other treatment can be done in individual patient having nasal blockage symptoms. The results are encouraging us to use the systematic questionnaire (SNOT -22) to estimate the severity of symptoms in daily clinical practice.

Key Words: Health related quality of life, nose outcome, septum, SNOT -22.

\section{Introduction}

Septoplasty is one of the most commonly performed surgery in ENT hospitals.The various indications for Septoplasty are nasal obstruction, crusting, rhinorrhoea , post nasal discharge, recurrent sinus pressure or pain, epistaxis ,headache, snoring, sleep apnoea are the indications for septoplasty ${ }^{(1)}$ In a questionnaire study done two years postoperatively, septoplasty relieved the symptoms well or excellently in 55\% and moderately in $27 \%$ of the 219 cases $^{(2 .}$ In another follow-up study of 35 cases, 9 months and at 9 years after the operation 55\% and $26 \%$ respectively, were subjectively free from obstruction ${ }^{(3)}$. Septoplasty sometimes results in only limited long lasting effect on nasal blockage ${ }^{(1)}$.

Several studies have investigated the effect of Septoplasty surgery on patient QOL .In a prospective study done by Arunachalam et al ${ }^{(4)} 200$ septoplasty patients filled preoperative and 6 week postoperative fairley nasal score ,Nottingham health profile , and General health questionnaire .Nasal obstruction improvement was seen in $74 \%$ and facial pain in $72 \%$ while postoperative improvement in nasal obstruction was independent of grade of the surgeon or concomitant lateral nasal wall surgery .However majority of the patients showed no change in generic NHP and GHQ.

In a study using NOSE score, $89 \%$ of the patients reported a subjective improvement in their nasal obstruction and especially younger patients seemed to benefit from septoplasty ${ }^{(5)}$. Calder and swan ${ }^{(6)}$ used generic glasgow benefit inventories in their study in which patients reported minimal improvement in their HRQOL following septal surgery.

A study at Helsinki general hospital ,Finland on patients operated for Septocollumeloplasty, in the department of otorhinolaryngology -Head and neck surgery where they analysed for 188 patients of which 126 patients by means of 15D and 22 items sino nasal outcome test (SNOT-22), where the questionnaire was given to the patients before the operation , on the operative day and 6 months postoperatively, only 126 patients answered all the questionnaire and that data was used for the comparison of pre and post operative results.

The present study was carried out to determine the effect of Septoplasty operation as measured by disease specific QOL questionnaire ie., SNOT- 22 questionnaire .

\section{Methodology:}

The study was conducted in the Department of ENT, Bangalore Medical College \& Research Institute, Bangalore. The study was carried out from September 2012 to December 2012, i.e. for a period of four months. The original study population consisted of 165 patients operated for septoplasty with other surgeries of nose and nasopharynx (Ex: FESS, Polypectomy, Rhinoplasty, Adenoidectomy, Turbinectomy, MMA etc). Out of 165 
cases only septoplasty cases operated during the period was 76 cases. So only 76 cases were considered initially for the pre-operative SNOT- 22 Questionnaire study and other cases were excluded from study group. Follow up was done at 1 week, 2 weeks, 4 weeks \& 8 weeks after surgery. SNOT- 22 Questionnaire study was done 8 weeks after surgery for analysis ( Post operative SNOT -22) After 8 weeks of septoplasty only 70 patients came for follow up \& 6 patients did not return for 8 weeks post surgical follow up. So for only 70 patients SNOT - 22 Questionnaire study was considered \& analysed. The patients were evaluated by pre-operative clinical status \& diagnostic nasal endoscopy, with \& without decongestion by Rhinoscopy. Septoplasty involved correction by mobilizing, straightening \& Reinserting the cartilaginous (and the bony) septum. All septoplasty operations was performed under local anaesthesia with sedation $(100 \%)$. Written informed consent for surgery was obtained from all study subjects.

The exclusion criteria of the study was:

$>$ Septoplasty in combination with sinus \& other surgeries

$>$ Rhinoplasty operation

$>$ Age less than 18 years and more than 55 years

$>$ Patients who did not return back for follow up after 8 weeks of surgery for various reasons

The operations was performed by 14 specialists and 3 senior residents. Mean operation time was 28 minutes +/- 12 minutes. Nasal splint was used in 10 cases (13.15\%). Next follow up visits was advised after 1 week, 2 weeks, 4 weeks \& 8 weeks after surgery. The 22 item Sino Nasal Outcomes Test (SNOT- 22) Questionnaire was given to the patient before the operation $\& 8$ weeks after the operation when the patient came for follow-up.

Out of 70 patients, 63 patients $(90 \%)$ were admitted in hospital one day before surgery \& 7 patients $(10 \%)$ came early morning on the day of surgery. SNOT -22 Questionnaire study was done on all these patients. All 70 patients (100\%) stayed overnight in the ward after surgery. Post operative nasal packing was done in all operations and for 67 patients nasal packing was removed on next day morning. For remaining 3 patients nasal packing was removed 2 days after surgery. All patients were advised local nasal decongestants for 5 days after pack removal..

The SNOT- 22 Questionnaire study has been used in studies on chronic Rhinosinusitis \& is also a useful tool in nasal septal surgery ${ }^{(7,8)}$. The SNOT -22 has 22 items and was recently reported to be valid \& easy to use. In the Questionnaire, patients rate each item from 0 (no problem) to 5 (problem as bad as it can be). The total maximum number of points in the SNOT-22 is thus $22 \times 5=110$ points. The patient is also asked to mark at maximum the five most important items. The scoring of SNOT-22 points is as mentioned below: $(0=$ no problem, $1=$ very mild problem, $2=$ mild or slight problem, $3=$ moderate problem, $4=$ severe problem, $5=$ problem as bad as it can be.)

The various nasal symptoms included were need to blow nose, sneezing, runny nose, nasal obstruction, cough, postnasal discharge etc. Where as QOL related symptoms included lack of good night's sleep, waking up tired, fatigue, reduced productivity and concentration, frustration etc.

In a septoplasty study done earlier, in which the SNOT -22 was used pre and 3 months postoperatively, the scores are $36.3 \& 19.3$ : the difference is $17.0^{(7)}$. The published scores of chronic sinusitis are $43.9 \& 31.3$ (post

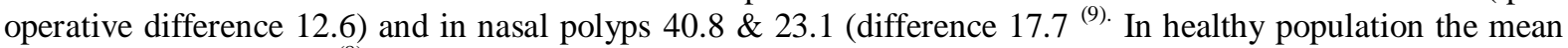
SNOT- 22 score is $9.3^{(8)}$. The minimally important difference i.e. the smallest change in the SNOT-22 score that can be detected by a patient is 8.9 points ${ }^{(9)}$.

Data Analysis \& Statistics of the study was done by a professional statistician. Statistical Analysis was done by paired T test.

\section{Results:}

Out of 70 patients, 48 patients (68.6\%) were males and 22 patients(31.4\%) were females. Mean age of patients was 29.1 (18-55) years. The mean operation time was $28 \pm 12$ Minutes. There was no restrictions or directions for the surgeon regarding the surgical procedure, use of post operative nasal pack or nasal splints.

Table 1: Showing Quality Of Life Related Symptoms In Pre And Post SNOT-22

\begin{tabular}{|l|l|l|l|}
\hline ITEMS & $\begin{array}{l}\text { PRE } \\
\text { Mean (SD) }\end{array}$ & $\begin{array}{l}\text { POST } \\
\text { Mean (SD) }\end{array}$ & P Value \\
\hline NEED TO BLOW NOSE & $1.614(1.33)$ & $0.857(0.82)$ & 0.0001 \\
\hline SNEEZING & $1.86(1.33)$ & $1.171(0.89)$ & 0.0001 \\
\hline RUNNY NOSE & $1.64(1.08)$ & $0.942(0.66)$ & 0.0001 \\
\hline
\end{tabular}


Septoplasty Outcome Using Snot- 22 Questionnaire Study

\begin{tabular}{|l|l|l|l|}
\hline NASAL OBSTRUCTION & $3.3(0.93)$ & $1.128(0.72)$ & 0.0001 \\
\hline LOSS OF SMELL OR TASTE & $0.8(0.88)$ & $0.428(0.55)$ & 0.0002 \\
\hline COUGH & $0.71(0.95)$ & $0.257(0.50)$ & 0.0003 \\
\hline POST-NASAL DISCHARGE & $0.986(0.97)$ & $0.228571(0.42)$ & 0.0001 \\
\hline THICK NASAL DISCHARGE & $2.1(1.02)$ & $0.942857(0.72)$ & 0.0001 \\
\hline EAR FULLNESS & $1.043(1.01)$ & $0.514286(0.65)$ & 0.0001 \\
\hline DIZZINESS & $0.886(0.94)$ & $0.814286(0.75)$ & 0.49 \\
\hline EAR PAIN & $0.79(0.98)$ & $0.657143(0.66)$ & 0.25 \\
\hline FACIAL PAIN/PRESSURE & $1.21(1.12)$ & $0.528571(0.7)$ & 0.0001 \\
\hline DIFFICULTY FALLING ASLEEP & $1.06(1.02)$ & $0.7(0.8)$ & 0.0001 \\
\hline WAKE UP AT NIGHT & $1.37(0.84)$ & $0.857143(0.55)$ & 0.0001 \\
\hline LACK OF GOOD NIGHT'S SLEEP & $1.243(0.88)$ & $0.814286(0.6)$ & 0.0001 \\
\hline WAKE UP TIRED & $1.13(0.85)$ & $0.8(0.6)$ & 0.0002 \\
\hline FATIGUE & $1 .(0.78)$ & $0.942857(0.63)$ & 0.41 \\
\hline REDUCED PRODUCTIVITY & $0.714(0.68)$ & $0.542857(0.58)$ & 0.013 \\
\hline REDUCED CONCENTRATION & $0.91(0.9)$ & $0.957143(0.77)$ & 0.59 \\
\hline FRUSTRATED/RESTLESS/IRRITABLE & $1.086(0.91)$ & $1.085714(0.76)$ & 1 \\
\hline SAD & $1.09(0.91)$ & $1.071429(0.77)$ & 0.9 \\
\hline EMBARRASSED & $0.56(0.67)$ & $0.771429(0.73)$ & 0.01 \\
\hline & & & \\
\hline
\end{tabular}

The mean age of all septoplasty patients was 29.1 years. The mean age of those patients who did not come for 8 weeks post surgical follow up was 23.5 years. The mean pre SNOT- 22 points were 26.93 (range 13 to 53). Post SNOT- 22 points was 17.01 (range 10 to 36$)$ and the difference was $9.92,(\mathrm{n}=70)$.

60 patients $(86 \%)$ who had mild to moderate symptoms with SNOT-22 scoring < 40 points and also young patients $<30$ years showed much improvement with the symptoms and relief from nasal obstruction after surgery.

Table 1 shows the mean values of pre and post SNOT- 22 items, and probability values. The items that showed significant improvement after surgery was need to blow nose, sneezing, running nose, nasal obstruction, loss of smell or taste, cough, post nasal discharge, thick nasal discharge, ear fullness, facial pain/pressure, difficulty in falling asleep, waking up at night, lack of good night's sleep, wake up tired, reduced productivity and embarrassed.

Fig 1.Pre And Post Op Means Of Five Most Important Items Complained By Patients With Differences Shown In The Figure Below.

Pre and Post OP Mean

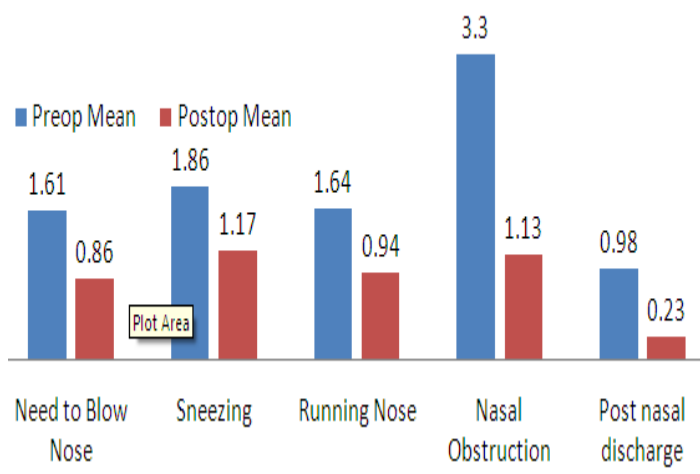




\section{Discussion}

By means of SNOT-22 Questionnaire study we were able to measure the effect of septoplasty. The 70 septoplasty operations done during the period from September 2012 to December 2012 was done in ENT Department of well recognised Bangalore Medical College. 8 weeks after the septoplasty, the 70 patients who came for follow up and the study as measured by SNOT - 22 Questionnaire showed significant improvement in the following items i.e need to blow nose, sneezing, running nose, nasal obstruction, loss of smell or taste, cough, post nasal discharge, facial pain/pressure, difficulty in falling asleep, waking up at night ,lack of good night's sleep, wake up tired, reduced productivity and embarrassed( $\mathrm{p}<0.005)$.

Also assessing was done with patients having more nasal symptoms \{ SNOT scoring points $>40(10$ patients) $\}$ and patients having SNOT scoring points $<40$ (60 patients). In patients having SNOT scoring points > 40 the mean preoperative scoring was 47.3 and mean postoperative scoring was 27.7 with a difference of 19.6 . There was much improvement in nasal symptoms but the patient was not completely cured of symptoms and also scoring pattern showing decreased Quality of life. In patients with SNOT scoring points $<40$ points the patients mean preoperative value was 24.28 and mean postoperative value was 15.23 with difference of 9.05 and patient was comparatively symptomatically better and with good quality of life. Majority of these patients were free from nasal obstruction.

Majority of septoplasty undergone patients showed marked improvement in disease specific symptoms, good quality of life, satisfaction \& decreased medication use. Severe pre operative nasal obstruction indicated a higher predicted improvement ${ }^{(10)}$. An improvement is defined as a reduction of $>/=2$ points on the SNOT-22 score.

Our SNOT-22 results was slightly better when compared to the expected results of septoplasty especially with some of the major symptoms like nasal obstruction which decreased from 3.29 to 1.12 , with a mean difference of 2.17 \& also with thick nasal discharge which decreased from 2.1 to 0.94 , with a mean difference of 1.16 . The mean total pre-SNOT score was $26.93 \&$ the total post SNOT score was 17.01 with a mean difference of 9.92 .

In the previous studies of septoplasty chronic rhino sinusitis and nasal polyposis, the decrease in symptom score was $17.0,12.6$, and 17.7 , respectively ${ }^{(7,9)}$. We conclude that our patients had significantly milder nasal symptoms than in the studies published earlier.

However the weakness of the study is lack of control group.

In healthy populations, the mean SNOT-22 score is 9.3 and in patients, the minimally important difference is 8.9 points. We were also able to recognise that in the few items of SNOT-22 (reduced concentration, frustrated/restless, irritable, sad, fatigue, ear pain and dizziness) the p values was not significant, suggesting decreasing HRQoL . It was also noted from the study that the more nasal symptoms the lower was the health related QoL but the disease specific nasal symptoms was markedly reduced after surgery. Thus careful diagnostics \& treatment of patients having nasal symptoms are important. so before doing the surgery \& for better outcome the severity of the symptoms should be assessed and the patients having only minor symptoms should not be operated on or at least the indication for septoplasty should be very carefully discussed with the patient for further management. So the results of this study encourage the use of a systematic questionnaire Ex: the SNOT- 22, for patients with nasal symptoms in daily clinical practice to estimate the severity of these symptoms.

\section{Conclusion:}

While analysing the outcome of this study, the study undertaken 8 weeks after septoplasty, the total SNOT-22 score was reduced, showing very well that septoplasty had reduced the nasal symptoms and also in some patients the HRQoL became poor in those patients in whom patients with SNOT scoring showed more symptoms and in patients where much improvement of symptoms was not seen after surgery and outcome was poor especially in older patients with more nasal symptoms. The classical case requiring corrective septoplasty is not a problem, but there is often a first line between the decision to operate or not to operate in clinical practice. Based on the results of this study, evaluation of the symptoms \& findings in the patients is essential in deciding whether the surgery or other treatment should be given to individuals having nasal symptoms. So our SNOT-22 Questionnaire study done pre operatively was very useful in knowing the severity of patient's nasal problems and helped us to evaluate in selecting the patient for septoplasty and also post surgical SNOT-22 study helped us in knowing the outcome regarding effectiveness of surgery. It also helped us in analysing the severity of symptoms in daily clinical practice. 


\section{References:}

[1] Van olphen AF (2008) The septum. In Scott- Brown's otorhinolaryngology. Head neck surg 2:1582-1588.

[2] Hytonen M, Blomgren K, lilja M et al (2006) How we do it: septoplasties under local anaesthetic are suitable for short stay surgery; the clinical outcomes. Clinical otolaryngol 31(1):64-68.

[3] Jessen M, Ivarson A, Malm L(1989) Nasal airway resistance and symptoms after functional septoplasty: comparison of findings at 9 months and 9 years. clinical otolaryngol allied Sci 14(3):231-234.

[4] Arunachalam PS, Kitcher E, Gray J et al(2001) Nasal septal surgery: evaluation of symptomatic and general health outcomes. Clin Otolaryngol Allied Sci 26(5):367-370.

[5] Gandomi B, Bayat A, Kazemei T(2010) Outcomes of septoplasty in young adults: the nasal obstruction septoplasty effectiveness study. Am J Otolaryngol 31(3):189-192.

[6] Calder NJ, Swan IR (2007) Outcomes of septal surgery, J laryngol Otol 121(11):1060-1063.

[7] Buckland JR, Thomas S, Harries PG(2003) Can the Sinonasal outcome test(SNOT-22) be used as a reliable outcome measure for successful septal surgery? Clinical Otolaryngol Allied Sci 28(1):43-47.

[8] Gillett S, Hopkins C, Slack R et al(2009) A pilot study of the SNOT-22 score in adults with no sinonasal disease. Clinical Otolaryngol 34(5):467-469.

[9] Hopkins C, Gillett S, Slack R et al (2009) Psychometric validity of the 22-item Sinonasal outcome Test.Clin Otolaryngol 34(5):447454.

[10] Stewart MG, Smith TL, weaver EM et al (2004) Outcomes after nasal septoplasty: results from the nasal obstuction Septoplasty effectiveness(NOSE) study. Otolaryngol Head neck Surg 130(3):283-290 